\title{
Malaria in the southern highlands of Tanzania: a review of hospital records
}

\author{
B.T.A. MAEGGA ${ }^{1 *}$, J. COX ${ }^{2} \&$ K.D. MALLEY ${ }^{1}$ \\ ${ }^{I}$ National Institute for Medical Research, Tukuyu Research Station, \\ P. O. Box 538, Tukuyu, Tanzania \\ ${ }^{2}$ London School of Hygiene and Tropical Medicine, London, UK
}

\begin{abstract}
Outpatient attendance and inpatient admission records were examined to assess malaria situation in ten hospitals in Mbeya and Iringa Regions in southern highlands of Tanzania for a period of fifteen years from 1986-2000. Generally, records were deficient, some hospitals with entire annual records missing for one or several years. However, malaria maintained a high profile as the leading cause of admissions and deaths among hospital attendees. Of the ten hospitals, seven recorded malaria as the leading cause of admissions for at least ten years; in three of these, it was also the top ranking cause of child death. Although the respective magnitude of malaria morbidity and mortality burdens was not directly correlated with altitude $(\mathrm{P}>0.5)$, three hospitals (Uwemba, Bulongwa and Ikonda) at above 2,000m, had relatively lower malaria morbidity and mortality burden compared to three worst affected facilities (Ilembula, Chimala and Mbozi) in the lower range of altitude, located in the flat plains. In conclusion, malaria is the major public health problem in the highlands districts of Mbeya and Iringa Regions in Tanzania that need the attention of health authorities and immediate intervention. However, more research is required to establish the true picture of the problem among the communities.
\end{abstract}

Key words: malaria, hospital, surveillance, highlands, Tanzania

\section{Introduction}

It is a universally accepted fact that malaria is a disease of public health importance globally. The disease causes the greatest suffering and impoverishment among poor people, particularly in Africa, Asia and Latin America (Bruce-Chwatt, 1993). About $90 \%$ of all malaria deaths in the world today occur in Africa south of the Sahara. This is because the majority of the infections in Africa are caused by Plasmodium falciparum, the most dangerous of the four human malaria parasites (AMR, 2003). In all malaria-endemic countries in Africa, $25-40 \%$ and $20-50 \%$ of all outpatients clinic visits and admissions, respectively, are for malaria (AMR, 2003). Malaria causes at least $20 \%$ of all deaths in children under 5 years of age in Africa. Children who survive malaria may suffer long-term consequences of the infection. Repeated episodes of fever and illness reduce appetite and restrict play, social interaction, and educational opportunities, thereby contributing to poor development. According to Murphy \& Breman (2001), an estimated $2 \%$ of children who recover from malaria infections affecting brain, suffer from learning impairments and disabilities due to brain damage.

The epidemiology of the disease in sub-Saharan Africa, to which Tanzania belongs, is heterogeneous. It varies from intense perennial, to highly seasonal low transmission for a relatively short time, to sporadic transmission experiencing epidemics in areas formally known to be malaria free. In epidemiological characteristic, areas of intense perennial transmission are classified as holoendemic, where malaria transmission is stable and occurs for more than 6 months per year, whereas areas of seasonal malaria are classified as hyper-endemic, where transmission occurs between 3 and 6 months, and hypoendemic where transmission occurs for less than 3 months (BruceChwatt, 1993).

In Tanzania, malaria is the leading cause of outpatient attendance, inpatient admission and hospital deaths (Kitua, 2003). It is envisioned that a larger proportion of deaths attributable to malaria occur outside the health facilities, at home, where no records are available (AMMP, 1997). Thus in actual fact, the magnitude of malaria attributable morbidity and mortality is difficult to assess through the existing health facility information gathering alone, but is recognized as much higher than is presented by hospital figures.

While many malaria epidemiological studies have been conducted in the north and especially north-eastern Tanzania (Cox et al., 1999; Mboera et al., 2002a, 2005), only a few similar works have

\footnotetext{
* Correspondence: B.T.A. Maegga; E-mail: maeggabta@africaonline.co.tz
} 
been done in the southern highlands zone in recent years (Mboera et al., 2001), which informs on the malaria situation in relation to the ongoing local ecological and global climatic changes (Cox et al., 1999). In this study we examined a 15 year clinical malaria data derived from 10 hospitals with outpatient and inpatient facilities in the southern highlands of Tanzania. These data provide an empirical basis for understanding the burden of malaria that will form basis for future research and design of appropriate interventions.

\section{Materials and Methods}

\section{Study area}

Ten voluntary agency hospitals were chosen within the southern highlands zone of Tanzania for their more consistent data collection records compared to government owned facilities. The hospitals included Isoko, Mbozi, Chimala, Igongwe and Itete in Mbeya region and Ilembula, Uwemba, Consolata Ikonda, Bulongwa and St John Lugalawa in Iringa region (Figure 1).

For each hospital, authorities were met for consultation and the objective of the study explained to them, prior to seeking their oral consent to examine the hospital records. Records were examined and data collected from the annual and monthly reports using an already prepared checklist. Records used were outpatient (OPD) attendance registers, inpatient (IPD) admissions registers and death records. Age-tallied cases and deaths $(<5$ years and $\geq 5$ years) of clinical malaria were compiled for each year in the ten hospitals. Not all diagnoses were microscopically confirmed. A Garmin Geographical Positioning System (GPS) unit was used to determine the coordinates and altitude for each hospital.

\section{Data analysis}

Information collected from the ten hospitals was collated and analysed for both OPD and IPD. Records of morbidity and mortality due to malaria were extracted, and recorded. MS Excel was used for data entry and processing. Data was analysed by year to determine any gradual changes in malaria cases during the 15 year period.
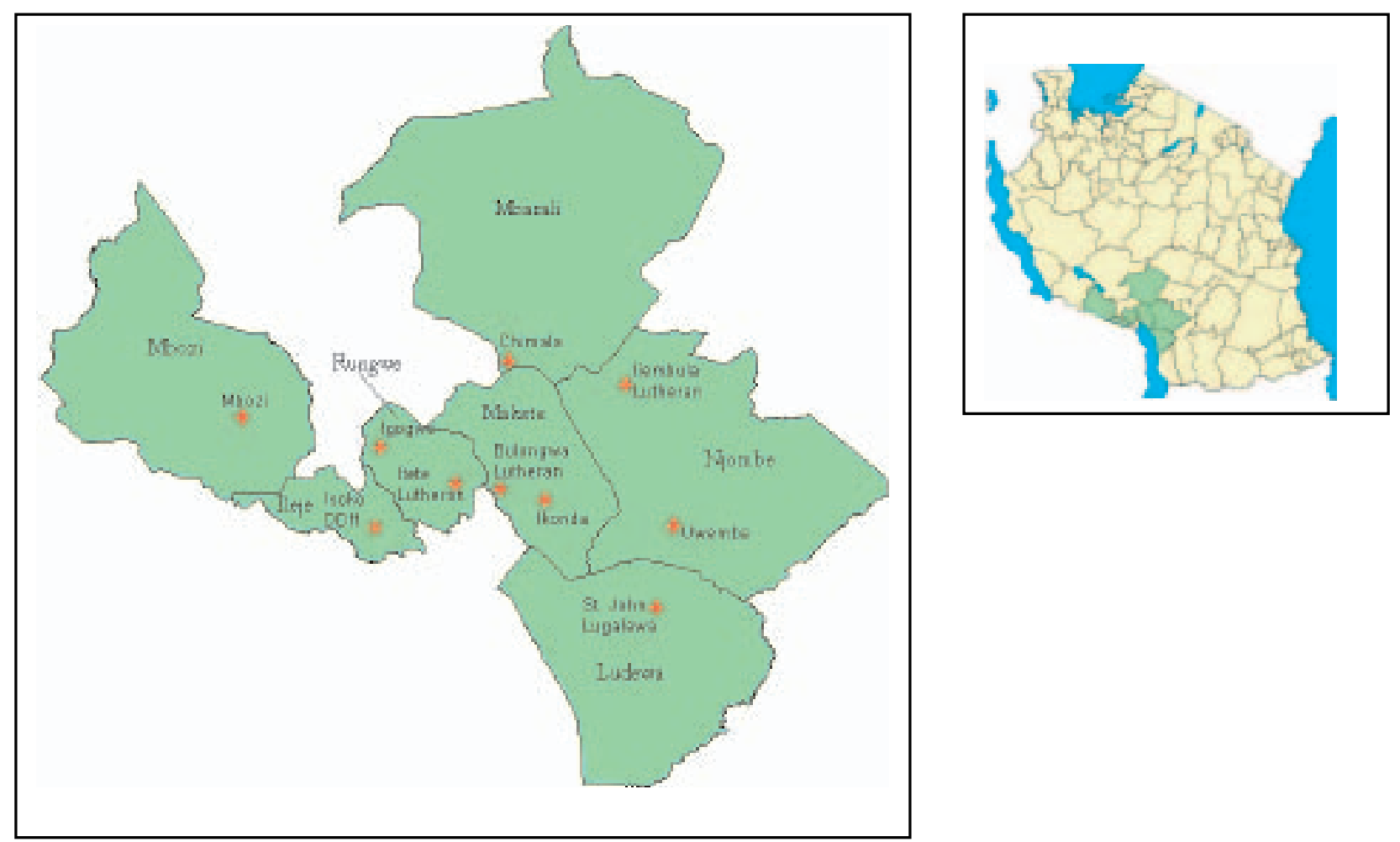

Figure 1: The location of the hospitals in Iringa and Mbeya Regions, Tanzania 


\section{Results}

Some hospitals lacked proper records for a portion of the period surveyed. The results of the OPD records showed a rather haphazard general annual fluctuation, where some hospitals like Isoko with quite high figures from 1987 to 1992, seemed to experience a significant drop in 1993, and continued declining albeit gradually until 1999 (Figure 2). It appears from these records, that Isoko, Lugalawa and Igogwe had high OPD attendance attributed to malaria between 1986 and 1993, when the magnitude dropped noticeably. On the other hand, there seemed to be a steady increase in OPD malaria cases in Mbozi between 1995 and 1998.

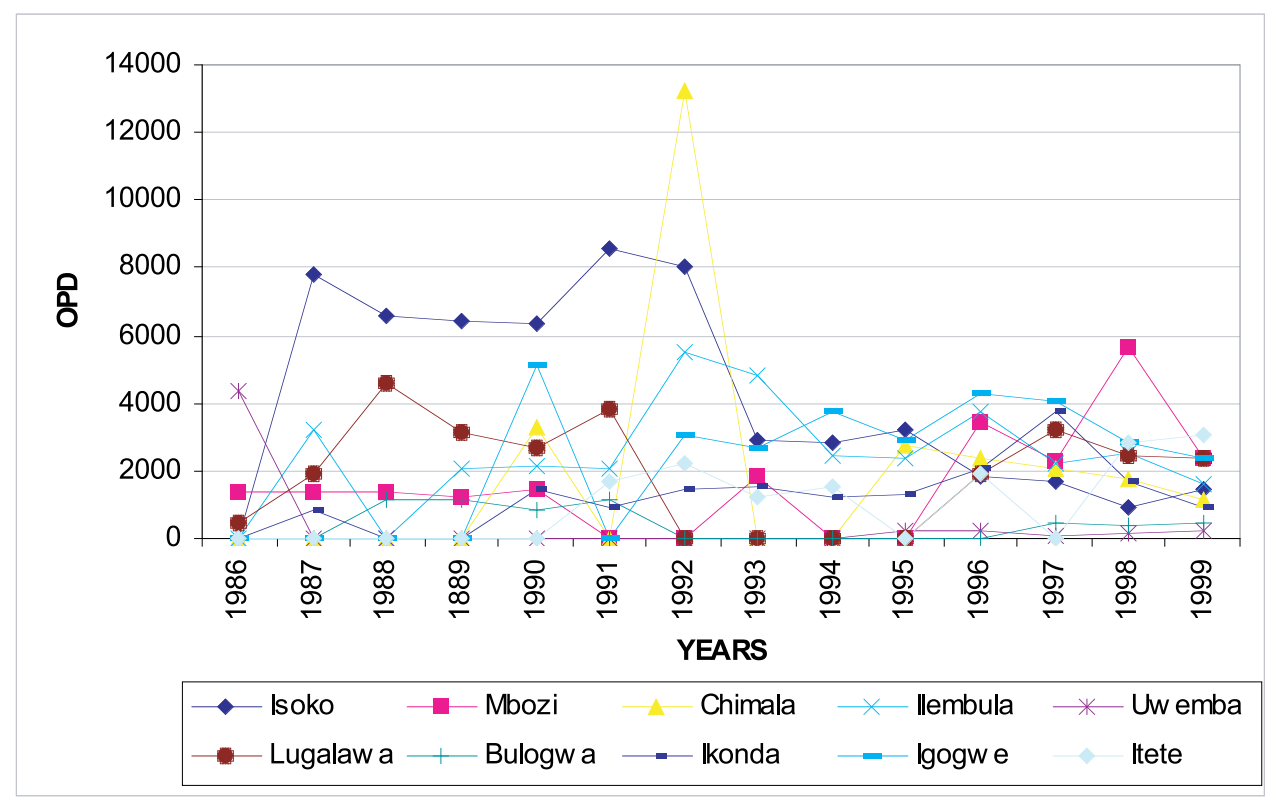

Figure 2: The pattern of outpatient malaria cases in hospitals in Iringa and Mbeya (1986 -2000)

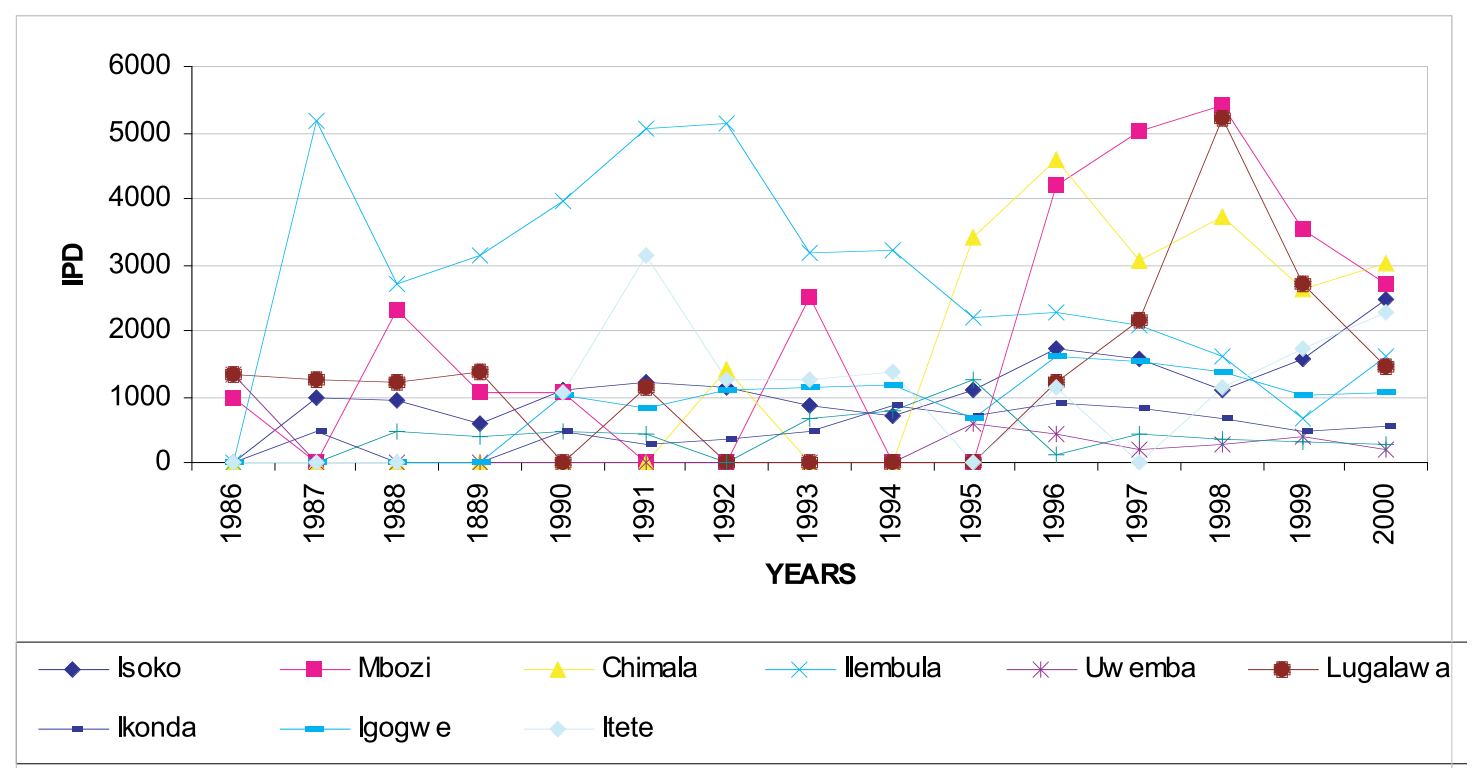

Figure 3: Pattern of admissions due to malaria in hospitals in Iringa and Mbeya (1986 2000) 
There was lack of data on admission in some health facilities and of the existing ones, there was an overall increase in admissions due to malaria from 1995 to 2000, in Isoko and Mbozi, but no definite pattern for the rest of the time. Lack of data was observed in the following cases (number of missing annual records is in parenthesis): Mbozi DDH (5), Chimala (8), Uwemba ((9), Itete (5), Bulongwa (4), and Ikonda (3). Such disparity of data collection makes it impossible to compare between facilities in the same year, or for the same hospital over several years. For Chimala and Lugalawa, the highest numbers of admissions were observed in 1998. Mbozi, Chimala and Lugalawa had high admissions among the ten facilities, while Ikonda and Uwemba had the lowest annual admissions attributed to malaria (Figure 2). The latter two are located in cool high altitude sites, above $2000 \mathrm{~m}$. Itete, Igogwe and Isoko hospitals were situated in mid-altitudes, in a mountainous terrain with relatively good natural drainage system, characterised by ridges and valleys. These had intermediate numbers of admission. From these records, the three showed intermediate values of malaria morbidity, between the high admission figures observed in Mbozi, Chimala and Lugalawa, and low ones in Uwemba and Ikonda, and to some extent, Bulongwa.

Malaria ranked as number one among the top ten leading causes of hospital admissions in seven of the ten hospitals visited. In three out of seven, it was also the top ranking cause of child death. In the other four, its rank fluctuated between the top, and $2-4$, in different years. In the remaining three facilities, it was a significant cause for admission, ranking 1-3 in Ikonda and Bulongwa, and 2- 6 in Uwemba. As a cause of death, it ranked 1-3 in Bulongwa, 2-4 in Uwemba and 2-7 in Ikonda.

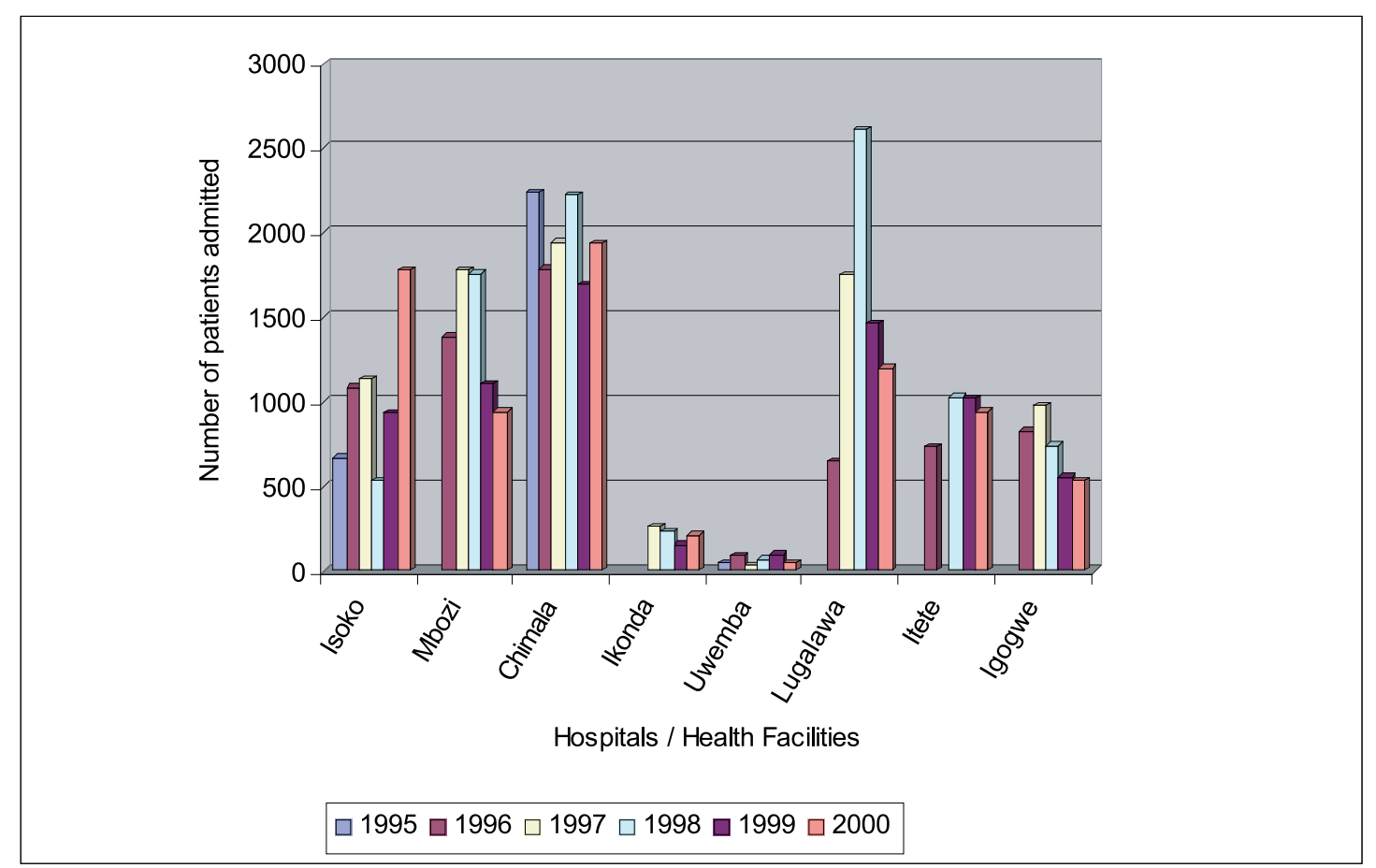

Figure 4: The number of admissions due to malaria in children $<5$ years in Iringa and Mbeya, 1995-2000 


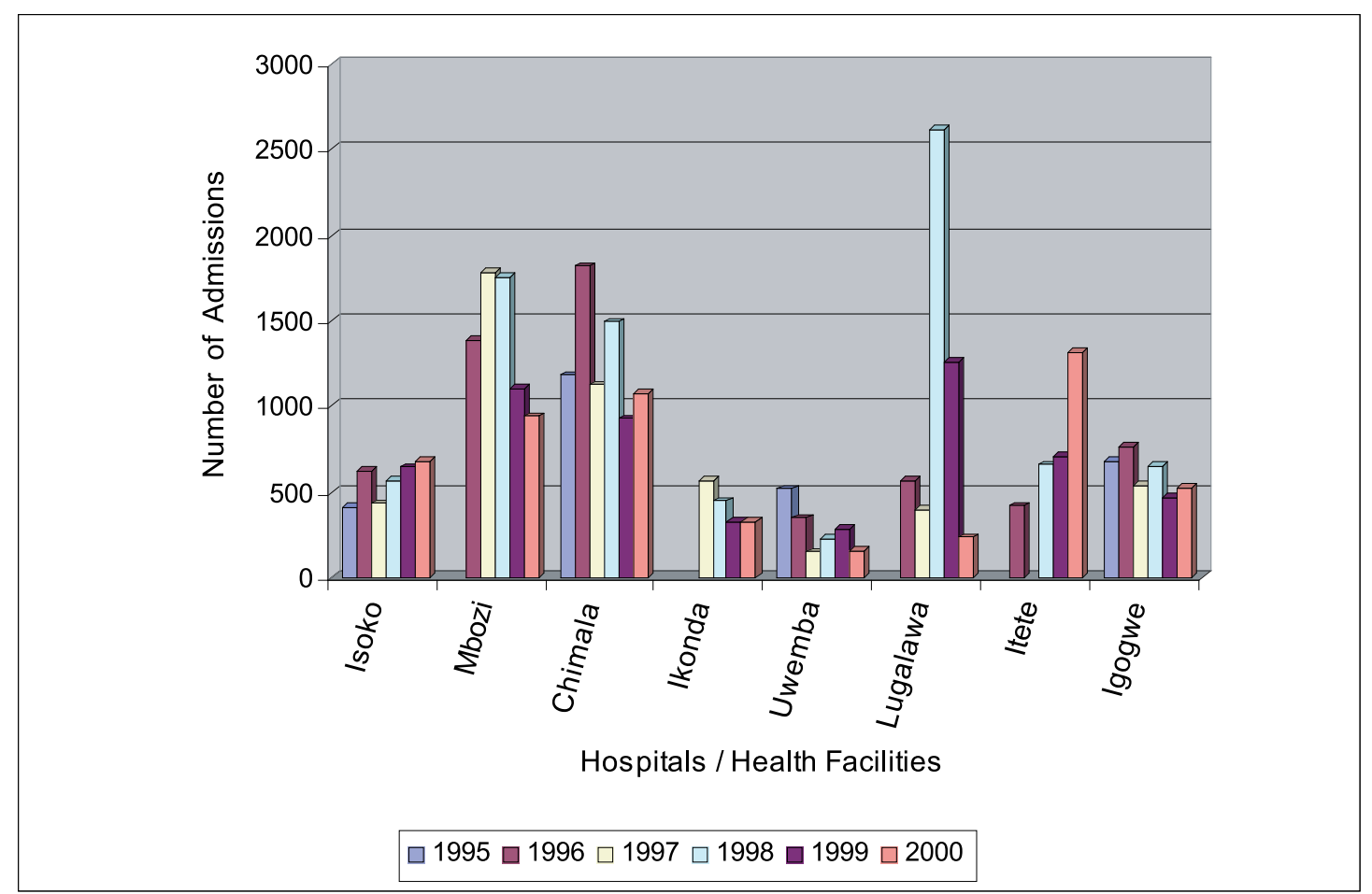

Figure 5: Malaria admission cases in 5 years in Iringa and Mbeya, 1995-2000

When the number of admission were examined in detail for both $<5$ years and $\geq 5$ years (Figure 4 and 5), Ikonda and Uwemba reported the lowest number of cases while Chimala and Lugalawa reported the highest number of cases between 1995 and 2000. In all hospitals, most of the admissions were observed during 1998. Hospitals in the higher altitudes $(>2000 \mathrm{~m})$ had much lower malaria burden compared to those in the 1000 to $1500 \mathrm{~m}$ range.

\section{Discussions}

Most of these areas, located at $>1500 \mathrm{~m}$ have previously been classified as having little or no malaria transmission, as a consequence of their topographical and associated meteorological conditions (Clyde, 1967). As one ascends into higher altitudes, temperature gradually decreases rendering the weather conditions unfavourable for malaria transmission. The areas were also formerly covered by indigenous dense forest, but a large portion of it has been cleared over the last few decades, with subsequent human habitation and intensification of rural subsistence agriculture. Both agricultural extension and tree felling for lumbering purposes and for domestic use have drastically devastated the forests reserves. Forest cutting for domestic use, especially as energy source and house construction is a perpetual problem all over, and no solution seems to be in sight. The radical ecological change of recent years may have directly or indirectly led to the increased malaria transmission as that observed in the Eastern Usambara Mountains (Matola et al., 1987).

In the high relief mountainous areas, it was observed that, the magnitude of malaria attributable morbidity and mortality showed a negative correlation with altitude where lower admissions and deaths were recorded in hospitals in altitudes between 2000 and $3000 \mathrm{~m}$. In Uwemba, Ikonda and Bulongwa hospitals, malaria cases were fewer in comparison with Chimala, Ilembula and Mbozi. The former three facilities lie at altitudes between 2000 $3000 \mathrm{~m}$, whereas the latter are in the plains, at altitudes between 1000 to $2000 \mathrm{~m}$. In the higher altitude trio, malaria was not considered as a top public health problem, but instead, other diseases like respiratory tract infections and HIV/AIDS were stated as the leading causes of admissions and deaths (data not shown). These comparisons, though relatively crude, considering the data deficiencies recognized, call for further investigation.

From observations of malaria records in the ten hospitals covered, the three worst affected hospitals are located in the lower part of the zone, whereas the 
least affected were in the higher part. The situation indicated by records at Ikonda, Uwemba and Bulongwa hospitals at above $2000 \mathrm{~m}$, and reporting much less malaria morbidity and mortality concurs well with observations made in the Usambara, Pare and Ngorongoro Mountains where cases of malaria were detected gradually less and less as one ascends to higher altitudes (Bødker et al., 1997; Mboera et al., 2001a, 2002a, 2005). The decline was attributed to unfavourable weather for building up large vector population, which is necessary to maintain intense transmission. Recently, parasitological studies in Iringa have shown a prevalence rate of $0-74.2 \%$ with higher parasite rates in villages at lower altitudes than higher altitudes. Malaria parasites were absent in children living at altitudes higher than 1900m (Mboera et al., 2001a).

Factors considered to be influencing the emerging situation regarding malaria transmission in the study area are: (i) the on-going global climatic changes, especially global warming seen in the rise in mean annual temperatures and local microclimatic conditions, which together, promote sporogony of malaria parasite; (ii) water resource development projects such as the large Mbarali (Kapunga) Rice irrigation scheme and (iii) the general deforestation of the natural vegetation as a result of increased human population pressure and expansion of rural agricultural activities. These factors have taken their toll on the local microclimatic conditions, thus contributing to increase of vector breeding distribution and intensity, with consequent increase in malaria transmission, for a longer duration than has been experienced previously in history. Due to improved communication means, in road transport which has made daily commuting from lowland to highland locations possible, there is increased importation of vectors and parasite from malaria endemic lowland sources to the highland localities (Cox et al., 1999).

As in any other region of the country, an increase in malaria incidence and prevalence over the past several decades is expected, and in this zone can be attributed to many factors including changing population structure, population movements from malaria holoendemic to hypoendemic or even originally malaria free areas, increased farming activities and immigration into new areas such as provided by the irrigation scheme of Mbarali
"Kapunga" rice farming in the Usangu plains, where malaria transmission is much higher compared to the highlands to the south and southwest. Lack of personal protective gear and absence of mosquito control schemes assure free spread of malaria from low to highlands. Traditionally, there is no protective means against man-mosquito contact and use of mosquito repellents is not common. In most of mainland Tanzania, proper environmental management for mosquito source breeding reduction has not been practiced postindependence, thus even in human created mosquito breeding sites near human dwellings, such as provided by mud brick making and road construction, one can expect unabated breeding, with consequent transmission of malaria. However, the importance of considering population growth and an increase in the rate of treatment seeking behaviour from health care facilities among the population can not be ignored. According to Hay et al. (2002), over the last 3 decades, there has been a high rate of increase in population size, resulting from high fertility rates and increasing child survival in much of sub-Saharan Africa.

From available records alone, the trend of malaria morbidity and mortality over time could not be assessed in some hospitals due to missing relevant data or incomplete annual records. Generally records were deficient, and in some hospitals, entire annual records were missing for several years. Completeness reporting in most of the facilities and districts in Tanzania is poor. Data quality is an important issue for a surveillance system and should be able to reflect the real situation in space and time (Gueye et al. 2005).

Although available data in this study is not adequate to make concrete conclusions, it nonetheless confirms that malaria is a major public health problem in a significant proportion of the southern highlands of Tanzania. Hospital records are biased at best, but in this case, they are used as a starting point. The fluctuation in the number of cases (both outpatients and inpatients) should be interpreted with care. Such changes could have been due to several factors such as changes in health information systems (including reporting criterion) or an intervention. For instance, in the mid-1990s, there was a change of the health management information system in the country (Mboera et al., 
2001). This is likely to have influenced the way recording and reporting of cases of diseases was done. However, such data are unreliable where completeness is lacking. Such situation is common in many facilities and districts of Tanzania (Mboera, 2004). There is need therefore to strengthen disease surveillance at all levels to have reliable data for planning interventions.

It is recommended that a systematic research involving facility and population based clinical and parasitological as well as transmission dynamics studies be conducted to establish an up to date database that can be used for monitoring malaria situation in the southern highlands, which have a complex relief geography. It is also essential to strengthen disease surveillance systems at facility level so that data collected is correct and credible, analysed adequately and promptly, and passed on for timely action. Such information is critical in devising control strategies at both facility and district level. Longitudinal ecological and transmission studies should be carried out to identify factors which maintain malaria transmission under different conditions, for at least one calendar year, in order to generate meaningful information for planning affordable control measures at district and community levels, and for predicting potential epidemic outbreaks in areas that are epidemic prone. Socio-economic impact of malaria in the southern highlands zone be studied in order to build sociopolitical awareness and solicit investment for malaria control at local level.

\section{Acknowledgements}

We are very grateful to all officers in charge and staff of all the ten hospitals visited for permission and assistance in accessing their annual reports, and for other useful information that enabled us to compile the data for this manuscript. Their cordial cooperation is greatly appreciated. We thank Benjamin Mayala for generating the maps. This study received a grant from the London School of Hygiene and Tropical Medicine to JC, for which we are very grateful.

\section{References}

AMMP (1997) Adult Morbidity and Mortality Project Report. End of Phase I. Ministry of
Health, Dar es Salaam, Tanzania

AMR (2003) Africa Malaria Report http://www.rbm.who.int/amd2004/amr2003/c h.htm. Accessed on August 5, 2005.

Bødker, R., Akida, J., Shayo, D., Kisinza, W., Msangeni, H.A., Pedersen, E.M. \& Lindsay, S.W. (2003) Relationship between altitude and intensity of malaria transmission in the Usambara Mountains, Tanzania. Journal of Medical Entomology 40, 706-717.

Bruce-Chwatt, L. (1993) Essential Malariology. William Heinemaun Medical Books Ltd. London.

Clyde, D.F. (1967) Malaria in Tanzania. London, Oxford University Press.

Cox, J., Craig, M. Sueur, D.L. \& Sharp, B. (1999) Mapping Malaria Risk in the Highlands of Africa. MARA/ HIMAL Technical Report, December 1999: 62-93.

Gueye, D., Senkoro, K.P. \& Rumisha, S.F. (2005) Monitoring and Evaluation of Integrated Disease Surveillance and Response in Tanzania, October-December 2003. Bethesda, MD: The Partners for Health Reformplus Project, Abt Associates Inc.

Hay, S.I., Noor, A.M., Simba, M., Busolo, M., Guyatt, H.L., Ochola, S.A. \& Snow, R.W. (2002) Clinical epidemiology of malaria in the highlands of western Kenya. Emerging Infectious Diseases 8, 543-548.

Kitua, A.Y. (2003). Malaria control in the context of integrated management of childhood illness in Tanzania: the challenges ahead. Tanzania Health Research Bulletin 5, 1-4.

Matola, Y.G., White, G.B. \& Magayuka, S.A. (1987) The changed pattern of malaria endemicity and transmission at Amani in the eastern Usambara mountains, north-eastern Tanzania. Journal of Tropical Medicine and Hygiene 90, 127- 134.

Mboera, L.E.G. (2004) Environmental and socioeconomic determinants of malaria epidemics in the highlands of Tanzania, Tanzania Health Research Bulletin 6, 11-17.

Mboera, L.E.G., Kamugisha, M.L., Malima, R.C., Mushi, A.K., Msuya, F.H., Masawe, T. \& Kitua, A.Y. (2002) Malaria prevalence and health seeking behaviour among communities of the lowlands and highlands of Gonja, Same 
District, north-eastern Tanzania. Tanzania Health Research Bulletin 4, 47-53.

Mboera, L.E.G. \& Kitua, A.Y. (2001) Malaria epidemics in Tanzania: An overview. African Journal of Health Sciences 8, 17-23.

Mboera, L.E.G., Rumisha, S.F., Magesa, S.M. \& Kitua, A.Y. (2001a) Utilisation of Health Management Information System in disease surveillance in Tanzania. Tanzania Health Research Bulletin 3, (2), 15-18.

Mboera, L.E.G., Malima, R.C., Mangesho, P.E., Senkoro, K.P. \& Mwingira, V. (2005) Malaria among the pastoral communities in the Ngorongoro Crater area, northern Tanzania. Tanzania Health Research Bulletin 7, 79-87.
Mboera, L.E.G., Kamugisha, M.L., Rumisha, S.F., Kisinza, W.N., Chuwa, G.J., Mkumbwike, B., Khihatura, A., Kadete, L., Mgohamwende, F., Kitua, A.Y. \& Molteni, F. (2001b) Malaria epidemiological studies in Iringa Rural District, Tanzania. Report submitted to the National Malaria Control Programme, Ministry of Health, Dar es Salaam, Tanzania. June 2001. Miller, L.F., Fields, R., Mmbuji, P.K.L., Posner, S., Mboera, L.E.G., Jimmerson, A., Senkoro, K.P., Rumisha, S.F., Shayo, E.H. \& Mwami, J.A. (2003) Situation Analysis of Infectious Disease Surveillance in two Districts in Tanzania, 2002. Bethesda, MD: The Partners for Health Reformsplus Project, Abt Associates Inc. 